\section{Evaluación in vitro del grado de microfiltración de tres cementos provisionales}

\section{In vitro evaluation of microfiltration degree of three temporary cements}

\begin{abstract}
Resumen
Objetivo: Evaluar el grado de microfiltración de tres cementos provisionales: IRM ${ }^{\circledR}$, OBTUR $^{\circledR}$ y CAVIT $^{\circledR}$ sometidos a envejecimiento artificial. Métodos: En 60 terceros molares, libres de caries, extraídos previa indicación terapéutica, fueron realizadas cavidades en la superficie oclusal con una apertura a cámara pulpar de $4 \mathrm{~mm} \times 4 \mathrm{~mm}$, tras irrigación con $\mathrm{NaClO}$ al 2,5\% y secado con algodón, los molares fueron divididos aleatoriamente en tres grupos ( $\mathrm{n}$ : 20) colocando en cada uno de ellos uno de los tres cementos. Los dientes fueron sometidos a termociclado con temperaturas de -5, 37 y 55 ${ }^{\circ} \mathrm{C}$; durante 900 ciclos continuos, tras lo cual fueron inmersas en azul de metileno por 24 horas, seccionados y analizados bajo estereomicroscopio para determinar el grado de microfiltración. Dos fragmentos de cada grupo fueron escogidos al azar y preparados para observación al microscopio electrónico de barrido (MEB). Resultados: El mayor grado de microfiltación fue observado en IRM. CAVIT $^{\circledR}$ y OBTUR $^{\circledR}$ presentaron un grado de microfiltración similar con menor microlfiltración, las observaciones realizadas al MEB corroboraron estos hallazgos. Conclusiones: El comportamiento de los cementos provisionales varía entre ellos, IRM ${ }^{\circledast}$ presentó un menor desempeño con mayor filtración, no existe diferencia entre CAVIT $^{\circledR}$ y OBTUR $^{\circledR}$ que presentaron un mejor desempeño.
\end{abstract}

Palabras clave: Adaptación marginal dental; Filtración dental; Restauración dental permanente.

\begin{abstract}
Objective: Evaluate the microfiltration degree of three temporary cements: IRM ${ }^{\circledR}$, OBTUR $^{\circledast}$ and CAVIT ${ }^{\circledR}$ subjected to artificial aging. Methods: In 60 third molars, without caries, extracted because of therapeutic indication, cavities were made in the occlusal surface with pulp chamber opening of $4 \mathrm{~mm} \times 4 \mathrm{~mm}$, after irrigation with $2.5 \% \mathrm{NaClO}$ and drying with cotton, the molars were randomly divided into three groups (n: 20) placing in each of them one of the three cements. The teeth were subjected to thermocycling at temperatures of $-5,37$ and $55^{\circ} \mathrm{C}$; during 900 continuous cycles, after which they were immersed in methylene blue for 24 hours, then sectioned and analyzed under a stereomicroscope to determine the microfiltration degree. Two fragments of each group were chosen randomly and prepared for scanning electron microscopy (SEM) observation. Results: The highest microfiltation degree was observed in IRM. CAVIT ${ }^{\circledR}$ and OBTUR ${ }^{\circledast}$ presented a similar degree of microfiltration with lower microfiltration, the observations made with the MEB corroborated these findings. Conclusions: The behavior of the provisional cements varies among them, IRM ${ }^{\circledast}$ presented a lower performance with higher filtration, there is no difference between $\mathrm{CAVIT}^{\circledR}$ and $\mathrm{OBTUR}^{\circledR}$ that presented better performance.
\end{abstract}

Keywords: Dental leakage; Dental marginal adaptation; Dental restoration, permanent.
ISSN: 1560-9111

\section{Artículo Original}

Paola Arrobo Espinosa 1,a, Alexander Victor Cruz Gallegos 2,a, Ana del Carmen Armas Vega 1,2,b, Alex Esteban Carrera Robalino ${ }^{2, a}$, Edisson López Ríos ${ }^{1, b}$

${ }^{1}$ Universidad Central del Ecuador.

2 Universidad Tecnológica Equinoccial.

a Odontólogo.

${ }^{\mathrm{b}} \mathrm{MSc}$. PhD.

Correspondencia:

Alexander Victor Cruz Gallegos

Correo electrónico: alexandercruz1993@ hotmail.com

Carlos Sánchez y Camilo Cien Fuegos N67-21. Quito, Ecuador.

Coautor:

Paola Arrobo Espinosa paola_arrobo@yahoo.com Ana del Carmen Armas Vega

ana_del_ec@yahoo.es

Alex Esteban Carrera Robalino alexcr199415@gmail.com

Edisson López Ríos edilopez33@hotmail.com

Conflicto de intereses: Los autores declaran no tener conflictos de interés.

Fuente de financiamiento: Autofinanciado

Fecha de recepción: 13/11/17

Fecha de aceptación: 26/04/18 


\section{Introducción}

El éxito del tratamiento endodóntico se encuentra estrechamente relacionado con la contaminación de los conductos radiculares ${ }^{1}$, intentando realizar el tratamiento en una cita para disminuir costos y tiempo ${ }^{2}$. En la práctica diaria muchas veces no es posible conseguir la finalización del tratamiento en una cita, teniendo que usar cementos provisionales como alternativa para alcanzar el sellado provisional e impedir la microfiltración y contaminación de los conductos radiculares ${ }^{3}$, productos como el IRM ${ }^{\circledR}$ a base de óxido de zinc y eugenol, OBTUR $^{\circledR}$ a base de óxido de zinc sin eugenol y CAVIT ${ }^{\circledR}$ también a base de óxido de zinc sin eugenol.

Una desventaja de los cementos provisionales es que tienden a deformarse ante la presencia de factores biológicos como la saliva ${ }^{4} \mathrm{y}$ la masticación ${ }^{5}$, e incluso ante procesos mecánicos del tipo traumas de oclusión, que se potencializan ante la flora bacteriana presente en la cavidad bucal favoreciendo la contaminación de los conductos radiculares ${ }^{6,7}$. Así su elección se convierte en un determinante del éxito a alcanzar ${ }^{8,9}$, sumado al hecho de que estos materiales como cementos provisionales, requieren cierta estabilidad en el tiempo ${ }^{4}$ por la cantidad de bacterianas presentes en la cavidad bucal ${ }^{9} \mathrm{y}$ exigen poseer cierta simplicidad en su remoción ${ }^{7,10}$.

La relación del fracaso endodóntico con la microfiltración de ciertos materiales ha estado estrechamente relacionada, cuando el tiempo del cemento provisional en boca excedía el lapso predispuesto por el fabricante ${ }^{9,11}$. La existencia de degeneración aumentaba con el subsecuente ingreso de fluidos orales contaminados a lo largo de la interfase diente-restauración ${ }^{12}$, lo que sucede con relativa frecuencia considerando que la cavidad bucal es un albergue de un gran porcentaje de microorganismos que cooperan con el ecosistema humano ${ }^{10}$, de ahí la importancia de seleccionar adecuadamente el material para sellado temporal ${ }^{13,14}$.

El presente estudio pretende evaluar el grado de microfiltración de tres cementos provisionales; IRM ${ }^{\oplus}, \mathrm{OB}_{-}$ TUR $^{\circledR}$ y CAVIT $^{\circledR}$ sometidos a envejecimiento artificial.

\section{Métodos}

Se realizó un estudio experimental, comparativo, in vitro, que fue sometido a evaluación por el comité de investigación de la Facultad de Odontología de la Universidad Central del Ecuador donde fue aprobado. Con una muestra de sesenta terceros molares superiores e inferiores extraídos previa indicación terapéutica y firma del consentimiento informado, estas piezas dentarias presentaron ápices abiertos o cerrados, libres de procesos cariosos, mantenidos en agua destilada y timol hasta su empleo, descartándose aquellos con fractura, restaurados o con fluorosis. En la superficie oclusal de cada uno de los dientes una vez limpios, mediante scaler ultrasónico (KAVO, Joinville, SC, Brasil), fueron preparadas cavidades de forma cuadrada de $4 \mathrm{~mm} \times 4 \mathrm{~mm}$, establecidas con una hoja de papel y un marcador permanente, mediante fresas de diamante redondas $\mathrm{N}^{\circ} 5$ (FAVA, Sao
Paulo, SP, Brasil) en alta rotación, intercambiadas cada cuatro cavidades, empleadas hasta producir acceso a cámara pulpar.

Los restos de tejido a nivel cameral fueron eliminados mediante $5 \mathrm{ml}$ de solución irrigadora de hipoclorito de sodio al 2,5\% (QUIMPAC, Guayaquil, Ecuador), seguida de suero fisiológico (LAMOSAN, Quito, Ecuador) aplicados mediante jeringa de insulina, realizándose el secado de las superficies con puntas de papel absorbente (DENTSPLY, México DF, México) y algodón estéril (MEDLINE, Guayaquil, Ecuador).

Sobre los ápices de las piezas dentales fue colocado ácido fosfórico al 37\% (FGM, Joinville, SC, Brasil) por 15 segundos, seguido de lavado, secado con jeringa triple colocando el sistema adhesivo Prime \& Bond (DENTSPLY, México DF, México) y fotopolimerizado por 20 segundos mediante lámpara de luz LED LITEX $696^{\circ}$ (DENTAMERICA, CA, USA). Cerrando los ápex con resina Alpha-Flow ${ }^{\circledR}$ (DENTAL TECHNOLOGIES Inc, Illinois, USA), fotopolimerizado por 20 segundos.

En las cavidades oclusales confeccionadas, fue colocada una bolita de algodón estéril a la entrada de los conductos radiculares cuidando de dejar $4 \mathrm{~mm}$ entre el borde cavo superficial de la cavidad y la torunda, medido a través de una sonda periodontal CP15 (Hu-Friendy, USA). Los 60 terceros molares fueron separados aleatoriamente en tres grupos (n: 20), obturando las cavidades preparadas con uno de los cementos provisionales probados; Grupo 1(G1): óxido de zinc-eugenol mejorado IRM ${ }^{\odot}$ (DENTSPLY, México DF, México), Grupo 2(G2): OBTUR ${ }^{\circledR}$ (MAQUIRA, Maringa, PR, Brasil) y Grupo 3 (G3): CAVIT ${ }^{\oplus}$ (3M ESPE, St. Paul, MN, USA). El IRM ${ }^{\oplus}$ fue preparado según las indicaciones del fabricante ${ }^{15}$ sobre loseta de vidrio y con la ayuda de un espátula metálica y colocado en la cavidad con un gutaperchero metálico, OBTUR ${ }^{\circledR}$ fue colocando previo secado de la cavidad con bolitas de algodón y aire, evitando producir su desecamiento empleando espátula de inserción y respetando los 5 minutos para fijación y 15 de secado ${ }^{16}$ y el CAVIT ${ }^{\circledast}$ fue colocado previo secado completo de la cavidad ${ }^{17}$.

Tras la colocación de los diferentes cementos provisionales según el grupo, los dientes fueron sometidos a baños térmicos durante 30 segundos a temperaturas de -5 , 37 y $55^{\circ} \mathrm{C}$, durante 900 ciclos, es decir 30 horas, simulando un mes en la cavidad bucal ${ }^{18}$. Concluido el proceso las superficies dentales fueron impermeabilizadas con esmalte de uñas dejando expuesta la interface diente-restauración, para luego introducir los dientes en una solución de azul de metileno al 4\% (QUIMPAC, Guayaquil, Ecuador) por 24 horas, tras lo cual, con un disco diamantado (FAVA, Sao Paulo, SP, Brasil) a baja velocidad cada diente fue dividido en dos fragmentos.

El análisis de cada fragmento fue realizado mediante microscopio estereoscópico (BIO OPTIC, Buenos Aires, Argentina) siguiendo metodología previamente establecida ${ }^{19}$, el grado de penetración fue establecido mediante valores numéricos considerando la penetración del tinte: 
$0=$ No hay penetración, $1=$ Penetración del tinte hasta la unión esmalte-dentina, $2=$ Penetración del tinte hasta la mitad de cámara pulpar y 3= Penetración del tinte más allá de la mitad de cámara pulpar. Los datos obtenidos considerando los dos fragmentos por diente y los valores numéricos correspondientes en las dos paredes de la cavidad fueron recolectados en tablas específicamente diseñadas y analizados mediante el paquete estadístico SPSS 22, mediante pruebas no paramétricas de Kruskal-Wallis y U Mann-Whitney, para probar si existen diferencias significativas en la microfiltración estimada para los tres cementos provisionales estudiados.

De forma complementaria se escogió dos dientes al azar de cada grupo y fueron analizados bajo microscopio electrónico de barrido (ZEISS, México DF, México), a una magnitud de 47x y 300x con un spot de 5,9. Las imágenes obtenidas sirvieron para complementar el análisis de las mediciones de microfiltración.

\section{Resultados}

El análisis descriptivo evidencio diferencias en cuanto al grado de microfiltración entre los cementos provisionales, donde IRM ${ }^{\circledR}$ fue quien obtuvo los mayores valores de microfiltración, en cuanto que OBTUR ${ }^{\circledR}$ y CAVIT $^{\circledR}$ presentaron valores muy similares entre ellos y con menor microfiltración. La prueba de rangos de Wilcoxon, ejecutada, evidenció diferencia en cuanto a la microfiltración estimada entre los dos fragmentos $(p=0,19)$ de cada diente. Tomando como base este resultado, fue realizada la prueba de Kruskal-Wallis, que determinó la existencia de diferencias significativas en la microfiltración valorada para los tres materiales $(p<0,001)$. Por lo que la prueba de U Mann-Whitney por pares fue realizada, evidenciando ella que el grupo donde se empleó IRM $^{\circledR}$ difiere significativamente de los otros dos grupos $(p<0,001)$ sin diferencias entre el grupo OBTUR ${ }^{\circledR}$ y CAVIT ${ }^{\circledR}$.

Las imágenes obtenidas bajo microscopio electrónico de barrido, correspondientes al G1, muestran la evidente brecha que se produce entre el material y las paredes cavitarias, lo que concuerda con los hallazgos estadísticos reportados (Figura 1). Al analizar la fotografía obtenida al emplear el G2 puede observarse una reducida brecha entre el material y las paredes dentarias (Figura 2) que lleva a pensar en el evidente me-

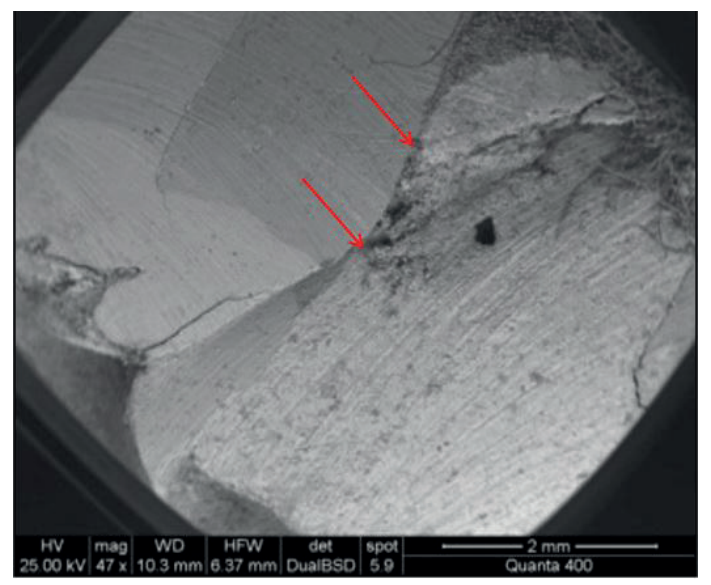

Figura 1. IRM ${ }^{\circledast}$ analizado a una magnitud de $47 x$ flecha señala interface diente- restauración con IRM ${ }^{\circledast}$

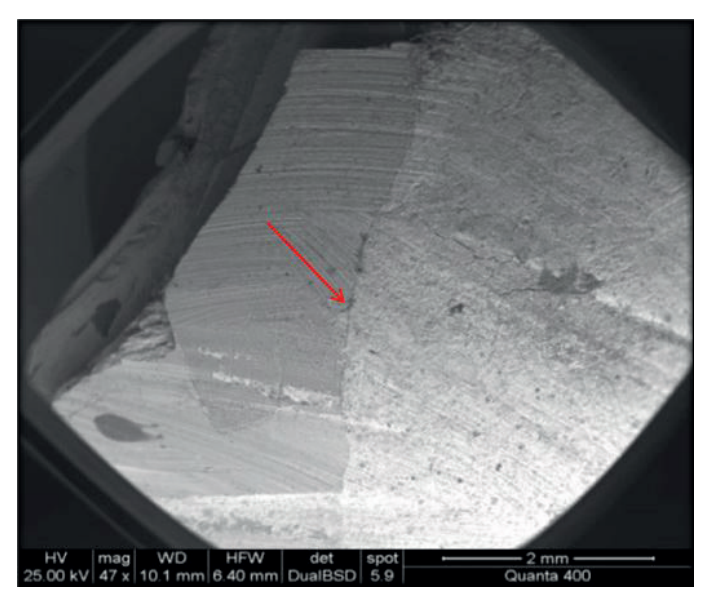

Figura 2. OBTUR ${ }^{\circledR}$ analizado a una magnitud de $47 x$ flecha señala interface diente-restauración con OBTUR ${ }^{\circledR}$

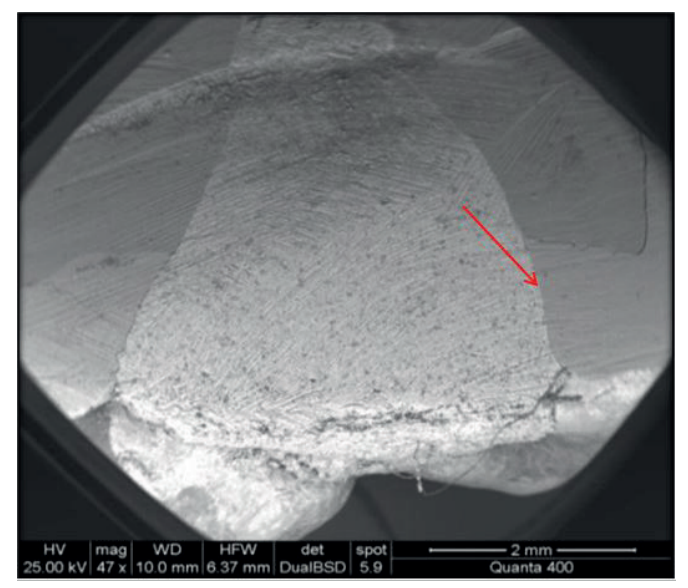

Figura 3. $\mathrm{CAVIT}^{\circledR}$ analizado a una magnitud de $47 \mathrm{x}$ flecha señala interface diente-restauración con $\mathrm{CAVIT}^{\circledR}$ 
jor desempeño del material, observación similar a las muestras del G3 (Figura 3).

\section{Discusión}

El estudio evidenció el pobre desempeño que tuvo IRM $^{\circledR}$ cuando se consideró la microfiltración, resultados que coinciden con estudios previos de Doveaux et al. ${ }^{20} \mathrm{y}$ Bergenholtz ${ }^{21}$, asociado a la composición misma del material y a la susceptibilidad que la dosificación y manipulación ${ }^{22,23}$ del material desencadena, sobre todo cuando un desafío de tiempo y temperatura existe, esto mencionados por Beach et al. ${ }^{22}$, Cruz et al. ${ }^{23}$, Srikumar et al. ${ }^{24} \mathrm{y}$ Widerman et al. ${ }^{25}$. Por su parte OBTUR ${ }^{\circledast}$ pese a ser un material también a base de óxido de zinc libre de eugenol, mostró mejores resultados en cuanto a la microfiltración, que podría explicarse por la presentación ya elaborada del material, como lo refirió Rodriguez et al. ${ }^{26}$ y su composición donde junto con el óxido de zinc, óxido de magnesio, sulfato de calcio anhidro y el sulfato de zinc monohidratado asegurarían el cierre hermético producido y verificándose con las fotografías obtenidas del microscopio electrónico de barrido, correspondiendo con el estudio de Hasen et al. ${ }^{27}$, destacando el hecho de la necesidad de colocar el material en una cantidad adecuada.

El CAVIT® ${ }^{\circledR}$ presentó en este estudio un desempeño semejante al OBTUR $^{\circledR}$ coincidiendo con el estudio de Tewari $e t$ al. ${ }^{28}$, asociando esto a la presencia en su composición de óxido de zinc, sulfato cálcico, sulfato de bario, sulfato de zinc, elementos que se presentan ya preparados lo cual aseguraría el desempeńo en cuanto al sellado mecánico, aun cuando con pobres propiedades adhesivas, como se evidenció en las fotografías al microscopio electrónico de barrido. Donde la brecha entre material, cualquiera que este sea, y tejido dentario, fue notoria de forma más clara y amplia cuando se empleó el IRM ${ }^{\oplus}$.

Si bien no existen diferencias significativas entre el tratamiento de una cita o en varias ${ }^{2}$, en la práctica clínica resulta frecuente postergarlos, aunque el desempeño de los cementos provisionales se haya mostrado adecuado en este estudio, estos tiempos no pueden excederse de 24 a 72 horas en boca como referido por Nageswar ${ }^{10}$, destacando que la prolongación de este periodo, puede desencadenar degradación del material independiente de cual fuera este ${ }^{5}$, asociado a su interacción con fluidos bucales, oclusión y las diferentes temperaturas, que a nivel bucal constituyen un verdadero desafío para el material en cuanto a mantener sus propiedades.

En los casos que resulta inminente la prolongación del tiempo de espera, los ionómeros de vidrio se muestran como alternativa, como lo refiere Imura et al. ${ }^{29}$, considerando siempre en esta elección la cantidad de tejido dental remanente, el esfuerzo mecánico de oclusión y los hábitos del paciente, mencionados también por Moon et al. ${ }^{30}$. Son necesarios nuevos estudios considerando variables que involucren espesores de tejido remanente así como prolongación de los tiempos.
En las condiciones en que este estudio fue ejecutado, puede afirmarse que IRM $^{\otimes}$ fue el cemento provisional que reportó mayor microfiltración, por otro lado, CAVIT $^{\circledR}$ y OBTUR ${ }^{\circledR}$ presentaron un comportamiento similar en cuanto a la microfiltración, siendo esta menor que el IRM ${ }^{\odot}$.

\section{Referencias bibliográficas}

1. Marshall FJ, Massler M. The sealing of Pulpples Teeth Evaluated with Radioisotopes. J Dent Med. 1961;16:172-184.

2. Manfredi M, Figini L, Gagliani M, Lodi G. Single versus multiple visits for endodontic treatment of permanent teeth. Cochrane Database Syst Rev. 2016;12:CD005296.

3. Leonardo MR. Endodoncia Tratamiento de conductos radiculares Principios Técnicos y Biológicos. 1 Vol. Sao Paulo: Artes Médicas Latinoamericana; 2005.

4. Canalda SC, Brau EA. Endodoncia Técnicas Clínicas y Bases Científicas. 2a ed. Barcelona: Masson; 2008.

5. Bottino MA. Endodoncia. 1a ed. Sao Paulo: Artes Médicas Latinoamericana; 2008.

6. Ingle J, Bakland L. Endodoncia. 4a ed. México D.F.: McGraw-Hill Interamericana; 1997.

7. Cova JL. Biomateriales Dentales. 2a ed. Mérida: Amolca; 2010.

8. Barcha D, Fortich R, Suarez L, Salvador O, Ramos I, González M. Ocurrencia de fracturas dentales ocasionadas por el uso de dos cementos temporales coltosol y óxido de zinc eugenol en molares. Rev Colomb investing odontol. 2009;1(1):38-45.

9. Soares J, Goldberg F. Endodoncia Técnicas y Fundamento. 1a ed. Buenos Aires: Panamericana S.A.; 2003. p.

10. Nageswar R. Endodoncia Avanzada. 1a ed. Caracas: Amolca; 2011.

11. Anderson J, McCabe F. Materiales de Aplicación Dental. 1a ed. Venezuela: Masson; 1988.

12. Balto H. An Assessment of microbial coronal leakage of temporary filling materials in endodontically treated teeth. J Endod. 2002;28(11):762-64.

13. Studervant RT, Heyman H, Edwar S. Arte y Ciencia de la Odontología. 5a ed. Madrid: Elsevier España; 2007.

14. Henostroza G. Caries Dental Principios y Procedimientos para el Diagnóstico. 1a ed. Lima: Multi-Impresos; 2007.

15. Dentsply Sirona. Para una práctica odontológica de mayor calidad, más rápida y más segura. Corporate dentsply sirona. [Consultado el 4 de diciembre 2017]. Accesible en: https://corporate.dentsplysirona.com/es.html

16. Maquira. Productos. Maquira dental Products. [Consultado el 4 de diciembre 2017]. Accesible en: http://www. maquira.com.br/

17. 3M ESPE. Productos. Fabricantes dentales 3M ESPE Fabricantes Dentales - 3M ESPE España. [Consultado el 4 de diciembre 2017]. Accesible en: http://solutions. productos3m.es/wps/portal/3M/es_ES/3M_ESPE/Dental-Manufacturers/ 
18. Lee YC, Yang SF, Hwang YF, Chueh LH, Chung KH. Microleakage of endodontic temporary restorative materials. J Endod. 1993;19(10):516-20.

19. Oppenheimer S, Rossenberg PA. Effect of temperature change on the sealing properties of cavit and cavit G. Surg Oral Med Oral Pathol. 1979;48(3):250-53.

20. Deveaux E, Hildelbert P, Neut C, Boniface B, Charles R. Bacterial microleakege of Cavit, IRM and TERM. Oral Surgery Med Oral Pathol. 1992;74(5):634-43.

21. Bergenholtz G. Endodoncia Diagnóstico y Tratamiento de la pulpa Dental. 1a ed. México D.F.: Manual Moderno; 2007.

22. Beach CW, Calhoun JC, Bramwell JD, Hutter JW, Miller GA. Clinical Evaluation of Bacterial Leakage of endodontic Temporary Filling Materials. J Endod. 1996;22(9):459-62.

23. Cruz EV, Shigetani Y, Ishikawa K, Kota K, Iwaku M, Goodis HE. A laboratory study of coronal microleakage using four temporary restorative materials. Int Endod J. 2002;35(4):315-20.
24. Srikumar GP, Varma KR, Shetty KH, Kumar P. Coronal microleakage with five different temporary restorative materials following walking bleach technique: An ex-vivo study. Contemp Clin Dent. 2012;3(4):421-26.

25. Widerman FH, Eaemes WB, Serene TP. The physical and biologic properties of Cavit. J Am Dent Assoc. 1971;82(2):378-82.

26. Rodríguez CI, Jácome JL, Perea LM. Estudio comparativo de filtración microbiana coronal con tres diferentes materiales de restauración provisional en dientes obturados con Guttaflow. Rev Odonto Mex. 2010;4(1):21-31.

27. Hansen SR, Montgomery S. Effect of restoration thickness on the sealing ability of TERM. J Endod. 1993;19(9):448-52.

28. Tewari S, Tewari S. Assessment of coronal microleakage in intermediately restored endodontic access cavities. Oral Surg Oral Med Oral Pathol Oral Radiol Endod. 2002;93(6):716-19.

29. Imura N, Otani SM, Campos MJ, Jardim EG, Zuolo ML. Bacterial penetration through temporary restorative materials in root-canal-treated teeth in vitro. Int Endod J. 1997;30(6):381-85.

30. Moon YH, Kim JR, Choi KK, Park SJ. The effect of thermocycling on the durability of dentin adhesive systems. J Korean Acad Conserv Dent. 2007;32(3):222-35. 
